
\title{
$\begin{array}{ll}\text { Research Square } & \begin{array}{l}\text { Preprints are preliminary reports that have not undergone peer review. } \\ \text { They should not be considered conclusive, used to inform clinical practice, } \\ \text { or referenced by the media as validated information. }\end{array}\end{array}$
}

\section{Acute effects of coffee on neurocognitive function: an electroencephalography network analysis}

\author{
Hayom Kim \\ Department of Neurology, Korea University Anam Hospital, Korea University College of Medicine \\ Sung Hoon Kang \\ Department of Neurology, Samsung Medical Center, Sungkyunkwan University School of Medicine \\ Soon Ho Kim \\ Laboratory of Computational Neurophysics, Brain Science Institute, Korea Institute of Science and Technology \\ Seong Hwan Kim \\ Department of Neurology, Korea University Anam Hospital, Korea University College of Medicine \\ Jihyeon Hwang \\ Department of Neurology, Korea University Anam Hospital, Korea University College of Medicine \\ Jae-Gyum Kim \\ Department of Neurology, Korea University Anam Hospital, Korea University College of Medicine \\ Kyungreem Han \\ Laboratory of Computational Neurophysics, Brain Science Institute, Korea Institute of Science and Technology \\ Jung Bin Kim ( $\sim$ kjbin80@korea.ac.kr) \\ Department of Neurology, Korea University Anam Hospital, Korea University College of Medicine
}

\section{Research Article}

Keywords: coffee, neurocognitive function, graph theoretical analysis, electroencephalography (EEG)

Posted Date: March 2nd, 2021

DOI: https://doi.org/10.21203/rs.3.rs-274504/v1

License: (c) (1) This work is licensed under a Creative Commons Attribution 4.0 International License. Read Full License

Version of Record: A version of this preprint was published at Scientific Reports on July 13th, 2021. See the published version at https://doi.org/10.1038/s41598-021-93849-7. 


\section{Abstract}

The purpose of this study was to identify the mechanisms underlying effects of coffee on cognition in the context of brain networks. Here we investigated functional connectivity before and after drinking coffee using graph theoretical analysis of electroencephalography (EEG). Twenty-one healthy adults voluntarily participated in this study. The neuropsychological tests were consecutively performed at the start of the EEG recording and 30 min after coffee consumption. Graph analyses were performed and compared before and after coffee consumption. Correlation analyses were conducted to assess the relationship between changes in graph measures and those in cognitive function tests. FC was reorganized toward more efficient network properties after coffee consumption. Performance in Digit Span tests and Trail Making Test Part B improved after coffee consumption, and the improved performance in executive function was correlated with changes in graph measures, reflecting a shift toward efficient network properties. The beneficial effects of coffee on cognitive function might be attributed to the reorganization of FC toward more efficient network properties. Based on our findings, the patterns of network reorganization could be used as quantitative markers to elucidate the mechanisms underlying the beneficial effects of coffee on cognition, especially executive function.

\section{Introduction}

Coffee is a widely used caffeinated beverage (International Coffee Organization, http://www.ico.org/prices/new-consumptiontable.pdf.), with more than 165 million $60-\mathrm{kg}$ bags consumed globally per year ${ }^{1}$. Potential beneficial health effects of coffee consumption have been reported, including prevention of cancer, cardiovascular disorders, diabetes, and Parkinson's disease ${ }^{2}$. Furthermore, given the expectation that coffee increases alertness and enhances psychomotor functioning, many people seek coffee to counteract fatigue, stay alert by warding off sleepiness, increase cognitive performance, and increase work efficiency ${ }^{3}$.

The stimulatory effects of coffee are mainly attributed to caffeine's roles in antagonizing adenosine $A_{1}$ and $A_{2 A}$ receptors, leading to disinhibition of excitatory neurotransmitter release and enhancement of dopamine transmission via $D_{2}$ receptor, respectively 4 . Although it is agreed that the acute effects of caffeine are due to its action as a central stimulant, inconsistent findings have been reported regarding the effects of coffee on higher cognitive functions, including working memory and executive functioning ${ }^{5}$. Some studies have shown beneficial effects of caffeine on cognitive functioning, including reaction times to cognitive tasks ${ }^{6}$, attention ${ }^{7}$, working memory ${ }^{8}$, and executive control ${ }^{9}$, whereas others reported no change after the use of caffeine ${ }^{10,11}$. Moreover, the expectancy of the stimulant effects of caffeine itself may have a role in the cognitive responses to caffeine ${ }^{11}$. Therefore, whether the beneficial effects of caffeine are derived from the direct enhancement of specific cognitive functions is unclear.

Integration of neural activities between different brain regions is required for physiological brain functioning. Therefore, analyzing functional connectivity (FC) between brain regions may provide more information than investigating activities of individual brain regions ${ }^{12,13}$. While most of the previous studies have evaluated the cognitive effects of caffeine mainly based on the results of neurocognitive tests, such as the Stroop test ${ }^{5}$, little attention has been paid to investigating the effects of caffeine on neurocognitive function in the context of FC. Recent advances in graph theoretical network analysis allow for the assessment of important information regarding the topological architecture of complex human brain networks ${ }^{14,15}$. Therefore, graph theoretical analysis could be an optimal framework for quantitatively characterizing network properties after coffee consumption and determining its effects on cognition.

To the best of our knowledge, no graph theoretical analysis has applied electroencephalography (EEG) data to explore the effects of caffeine on FC. Here, we compared the properties of FC before and after coffee consumption to analyze the acute effects of caffeine on the brain network and its impact on neurocognitive function using graph theoretical analysis of EEG data. We hypothesized that caffeine might improve neurocognitive function by shifting the FC of the brain to a more efficient state.

\section{Methods And Materials}

Participants. Twenty-one healthy volunteers (11 women; $31.4 \pm 3.9$ years; $17.0 \pm 1.4$ years of education) who had no neurologic, psychiatric, chronic systemic disorders, or medical conditions that could affect the EEG results were included in this study. All participants were requested to abstain from drinking beverages containing caffeine and from the use of any psychoactive 
substances or medication for at least $24 \mathrm{~h}$ prior to the EEG and neurocognitive studies ${ }^{16}$. All subjects were fully informed of the nature and possible risks of this study. Written informed consent was obtained from all subjects prior to study enrollment. The study followed the ethical guidelines of the Declaration of Helsinki and was approved by the local ethics committee at Korea University Anam Hospital (No. 2019AN0418).

Neurocognitive function tests and caffeine intake. Global neurocognitive function was assessed using the Mini-Mental State Examination (MMSE) at least $24 \mathrm{~h}$ after cessation of coffee consumption in all participants. The neuropsychological tests were selected to evaluate the acute effects of caffeine on performance in multiple neurocognitive domains. The assessed domains and the tests were as follows: (1) attention and working memory-Digit Span Forward (up to nine digits) and Backward (up to eight digits) tests ${ }^{17}$, Target Detection Task using tapping; (2) executive function- Trail Making Test Part B (time to complete the tests) ${ }^{18}$; and (3) memory-Short-term memory recall task (two learning trials of five words) and delayed recall (after 5 min). All neuropsychological tests were performed at the start of the EEG recording and $30 \mathrm{~min}$ after consumption of canned coffee ${ }^{19}$ using the same tests with a different set of contents. A commercial canned coffee (let us be ${ }^{\mathrm{TM}}$, Lotte Chilsung Beverage), which has the largest market share in Korea, was used for caffeine intake. One can of the canned coffee contains $160 \mathrm{~mL}$ and $67 \mathrm{mg}$ of caffeine.

Electroencephalography recording. The EEG examination was performed twice, once at baseline and then again 30 min after the participants drank the canned coffee treatment ${ }^{19}$, using a 32-channel recording system (Grass Technologies, Quincy, MA) with electrodes placed according to the international 10-20 system. The EEG was recorded for $1 \mathrm{~h}$ in the waking-relaxed and eyesclosed conditions. EEG data were sampled at $200 \mathrm{~Hz}$, and the bandpass filter was set between 0.1 and $70 \mathrm{~Hz}$.

Since the neuropsychological tests evaluating different domains were performed in succession, resting-state EEG data were used for analysis to avoid mixed effects of different domain-specific functional networks in this study. Ten non-consecutive resting state 2-s epochs for each participant were carefully reviewed and selected by two board-certified neurologists according to the following protocol: (1) presence of continuous physiological alpha activity with voltage maximum in posterior regions; (2) absence of artifacts, epileptiform discharges, and other nonstationary elements; and (3) absence of patterns indicating drowsiness or arousal.

Graph theoretical and statistical analyses. Resting-state FC was evaluated by coherence, which reflects the level of functional signal communication between different regions of the brain ${ }^{20}$. The coherence is defined as $\operatorname{coH}_{x y}=k_{x y}{ }^{2}(f)=\left|K_{x y}(f)\right|^{2}=\frac{\left|S_{x y}(f)\right|^{2}}{S_{x x}\left(f S_{y y}(f)\right.}$, where $S_{x y}(f)$ is the cross-spectral density between $x$ and $y$, and $S x x(f)$ and $S y y(f)$ are the auto-spectral densities of $x$ and $y$, respectively. K represents the coherency function. $|S|$ denotes the modulus of $S$. The coherence value ranges between 0 and 1 with 0 denoting no statistical relationship and 1 being full coherence ${ }^{20}$. In addition, the phase lag index (PLI) was used to measure phase synchronization between all pairs of $19 \mathrm{EEG}$ channels ${ }^{21,22}$. The PLI is defined as $P L I=\left|<\operatorname{sign}\left[\Delta \varphi\left(t_{k}\right)\right]>\right|$, where $\Delta \varphi\left(t_{k}\right)$ is the phase difference of time series $t_{k}(k=1, \ldots, N)$. It ranges between 0 and $1-0$ indicates either no coupling or phase difference centered around $0 \bmod \pi$, while 1 indicates perfect phase synchrony as a value of $\Delta \varphi$ different from $0 \bmod \pi$. Epochs were then bandpass filtered into the following frequency bands: delta $(0.1-4 \mathrm{~Hz})$, theta $(4-8 \mathrm{~Hz})$, alpha $(8-13 \mathrm{~Hz})$, beta $(13-30 \mathrm{~Hz})$, and gamma $(30-50 \mathrm{~Hz})$. Subsequent analyses were performed separately for each band. Network properties were characterized using a weighted undirected network model of graph theoretical analysis in order to avoid the arbitrariness of threshold selection for producing an adjacency matrix and to preserve the continuous nature of the correlated information ${ }^{23}$. Graph measures (average degree, average strength, radius, diameter, characteristic path length, global and local efficiency, clustering coefficient, transitivity, modularity, assortativity, and small-worldness) were computed using the Brain Connectivity Toolbox (http://www.brainconnectivity-toolbox.net) and BRAPH toolbox (http://braph.org) working on MATLAB R2019b (MathWorks, Natick, MA, USA) 23,24. $\mathrm{PLI}$ analysis and visualization were performed using tailored Python scripts and the MNE-Python package (version 0.22.0) ${ }^{25}$.

Graph measures were compared before and after consumption of canned coffee using non-parametric tests with 1,000 permutations. Statistical significance was set at $\mathrm{P}<0.05$ and corrected for multiple comparisons using false discovery rate (FDR). Differences in the results of neuropsychological tests before and after consumption of canned coffee were compared using paired $t$-test. Since the ranges of the graph measures' values differed from each other, the degree of change was normalized, and then the 
correlation between the changes of neuropsychological tests results and those of graph measures was analyzed using the normalized changed values (Pearson's correlation, $\mathrm{P}<0.05$ ).

\section{Results}

Neuropsychological tests. The results of the neuropsychological tests are detailed in Table 1. All participants had an MMSE score of 30. Performance in the Digit Span Forward ( $8.5 \pm 0.8$ digits vs. $8.9 \pm 0.2$ digits, $P=0.025)$ and Backward tests $(6.2 \pm 1.8$ digits vs. $7.3 \pm 1.1$ digits, $P=0.001)$ improved after coffee consumption relative to baseline. There were no errors in the target detection task using the tapping test, Trail Making Test Part B, and short-term and delayed memory recall tests before and after coffee consumption. Compared to baseline, performance in the Trail Making Test Part B improved after coffee consumption (5.8 $\pm 1.4 \mathrm{~s}$ vs. $4.9 \pm 1.2 \mathrm{~s}, \mathrm{P}=0.002$ ). Individual changes in performance in the Trail Making Test Part $\mathrm{B}$ after coffee drinking are presented in Figure 1 .

Table 1. Results of neuropsychological tests.

\begin{tabular}{|c|c|c|c|}
\hline & Baseline & After coffee consumption & P value \\
\hline $\begin{array}{c}<\text { Global function }> \\
\text { Mini-mental status examination } \\
\text { (correct / } 30 \text { items) }\end{array}$ & 30 & 30 & - \\
\hline $\begin{array}{c}<\text { Attention and working memory }> \\
\text { Digit Span Test forward }\end{array}$ & & $8.95 \pm 0.22$ & 0.025 \\
\hline $\begin{array}{c}\text { Digit Span Test backward } \\
\text { (correct / } 8 \text { digits) }\end{array}$ & $6.24 \pm 1.76$ & $7.29 \pm 1.10$ & 0.001 \\
\hline $\begin{array}{c}\text { Target Detection Task } \\
\text { (correct / } 11 \text { syllable targets) }\end{array}$ & 11 & 11 & - \\
\hline $\begin{array}{c}<\text { Executive function }> \\
\text { Trail Making Test Part B (s) }\end{array}$ & $5.80 \pm 1.41$ & $4.87 \pm 1.17$ & 0.002 \\
\hline $\begin{array}{c}<\text { Memory }> \\
\text { Short-term memory recall } \\
\text { (correct / } 5 \text { words) }\end{array}$ & 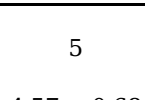 & 101 & - \\
\hline Delayed recall (correct / 5 words) & $4.57 \pm 0.68$ & $4.81 \pm 0.40$ & 0.135 \\
\hline
\end{tabular}

Values represent mean \pm standard deviation.

Graph theoretical analyses. FC in terms of coherence is represented with adjacent matrices, connectivity circles, and brain topologies in Figure 2A-C. PLIs are presented using the connectivity circles in Figure 3. FC was enhanced in all frequency bands for both methods, and similar patterns were found. The delta and gamma bands, in particular, were strongly increased in both coherence and PLI. By extracting the ten most highly increased links, the ten channels with the highest increases are shown in Figure 4, showing the regions most involved in the increase of FC. Comparisons of global graph measures between the conditions are detailed in Table 2.

Table 2. Comparisons of global graph measures between baseline and after coffee consumption. 


\begin{tabular}{|c|c|c|c|c|c|c|c|c|c|c|c|c|c|c|c|}
\hline \multirow[t]{2}{*}{$\begin{array}{l}\text { Graph } \\
\text { measures }\end{array}$} & Baseline & Coffee & $\begin{array}{c}\mathrm{P} \\
\text { value }\end{array}$ & Baseline & Coffee & $\begin{array}{c}\mathrm{P} \\
\text { value }\end{array}$ & Baseline & Coffee & $\begin{array}{c}P \\
\text { value }\end{array}$ & Baseline & Coffee & $\begin{array}{c}\mathrm{P} \\
\text { value }\end{array}$ & Baseline & Coffee & $\begin{array}{c}\mathrm{P} \\
\text { value }\end{array}$ \\
\hline & \multicolumn{3}{|c|}{ Delta } & \multicolumn{3}{|c|}{ Theta } & \multicolumn{3}{|c|}{ Alpha } & \multicolumn{3}{|c|}{ Beta } & \multicolumn{3}{|c|}{ Gamma } \\
\hline $\begin{array}{l}\begin{array}{l}\text { Average } \\
\text { degree }\end{array} \\
\end{array}$ & 13.920 & 15.058 & 0.001 & 12.005 & 12.988 & 0.013 & 10.782 & 11.378 & 0.043 & 12.145 & 13.033 & 0.027 & 14.852 & 15.649 & 0.061 \\
\hline $\begin{array}{l}\text { Average } \\
\text { strength }\end{array}$ & 9.253 & 11.333 & 0.001 & 6.175 & 7.338 & 0.010 & 5.037 & 5.434 & 0.021 & 5.924 & 6.376 & 0.125 & 9.453 & 10.685 & 0.012 \\
\hline Radius & 5.885 & 6.120 & 0.810 & 13.820 & 6.191 & 0.923 & 5.481 & 5.760 & 0.218 & 7.162 & 5.194 & 0.112 & 4.414 & 4.336 & 0.915 \\
\hline Diameter & 10.702 & 9.697 & 0.586 & 17.776 & 10.809 & 0.948 & 9.598 & 9.773 & 0.720 & 11.061 & 9.066 & 0.459 & 7.952 & 7.544 & 0.510 \\
\hline $\begin{array}{l}\text { Characteristic } \\
\text { path length }\end{array}$ & 2.799 & 2.377 & 0.218 & 4.071 & 3.056 & 0.183 & 3.520 & 3.377 & 0.228 & 3.598 & 3.116 & 0.054 & 2.449 & 2.182 & 0.066 \\
\hline $\begin{array}{l}\text { Global } \\
\text { efficiency }\end{array}$ & 0.568 & 0.659 & 0.004 & 0.444 & 0.488 & 0.015 & 0.397 & 0.412 & 0.054 & 0.428 & 0.445 & 0.108 & 0.580 & 0.640 & 0.029 \\
\hline $\begin{array}{l}\text { Local } \\
\text { efficiency }\end{array}$ & 1.718 & 2.255 & 0.001 & 1.057 & 1.267 & 0.019 & 0.826 & 0.884 & 0.026 & 0.945 & 1.004 & 0.208 & 1.696 & 1.958 & 0.049 \\
\hline $\begin{array}{l}\text { Clustering } \\
\text { coefficient }\end{array}$ & 0.560 & 0.670 & 0.002 & 0.380 & 0.439 & 0.035 & 0.328 & 0.341 & 0.197 & 0.357 & 0.372 & 0.277 & 0.542 & 0.605 & 0.052 \\
\hline Transitivity & 0.913 & 1.083 & 0.002 & 0.595 & 0.699 & 0.022 & 0.500 & 0.525 & 0.140 & 0.554 & 0.581 & 0.208 & 0.858 & 0.951 & 0.046 \\
\hline Modularity & 0.063 & 0.026 & 0.010 & 0.188 & 0.137 & 0.001 & 0.258 & 0.241 & 0.329 & 0.195 & 0.171 & 0.101 & 0.070 & 0.036 & 0.011 \\
\hline Assortativity & 0.111 & 0.050 & 0.188 & 0.160 & 0.077 & 0.105 & 0.156 & 0.123 & 0.223 & 0.122 & 0.080 & 0.116 & 0.025 & 0.019 & 0.202 \\
\hline $\begin{array}{l}\text { Small- } \\
\text { worldness }\end{array}$ & 1.042 & 1.233 & 0.612 & 0.855 & 1.250 & 0.289 & 0.891 & 1.054 & 0.460 & 0.868 & 0.945 & 0.364 & 0.927 & 0.901 & 0.166 \\
\hline
\end{tabular}

Values represent the mean.

Compared to baseline, the relative ratio of global graph measures after coffee consumption is presented in Figure 5A. Average degree (except in gamma band), average strength (except in beta band), global efficiency (except in alpha and beta bands), and local efficiency (except in beta band) increased after coffee consumption relative to baseline in most frequency bands (FDRcorrected $\mathrm{P}<0.05)$. The clustering coefficient in delta and theta bands, as well as transitivity in the delta, theta, and gamma bands, increased after coffee consumption relative to baseline (FDR-corrected $\mathrm{P}<0.05$ ). Compared to baseline, modularity in the delta, theta, and gamma bands decreased after coffee consumption (FDR-corrected $\mathrm{P}<0.05$ ). Significant differences in the nodal measures (degree, strength, global efficiency, local efficiency, and clustering coefficient) between the conditions were prominent mainly in the fronto-centro-parietal regions, especially in the delta and theta bands (Figure 5B).

Correlation analyses. The degree of improved performance in Trail Making Test Part B after coffee consumption was negatively correlated with diameter in the alpha band $(r=-0.657, P=0.002)$ and assortativity in the beta band $(r=-0.488, P=0.029)$, whereas it was positively correlated with small-worldness in the alpha band $(r=0.627, P=0.004$; Figure 6$)$. There was no relationship between the results of other neuropsychological tests and changes in graph measures.

\section{Discussion}

We investigated the acute effects of caffeine on neurocognition and EEG FC in healthy adults. The major findings were as follows: 1) the property of EEG FC was reorganized toward a more efficient network after coffee consumption relative to baseline, 2) Performance in the Digit Span tests and Trail Making Test Part B was improved after coffee consumption, and 3) improved performance in the Trail Making Test Part B after coffee consumption was correlated with changes in graph measures reflecting a shift toward efficient network property.

The human brain is considered to be a large-scale complex network and has properties of efficient small-world networks that refer to locally well-connected clusters and efficient global connections ${ }^{23,26}$. The properties of small-world networks are known to enable higher rates of information processing and learning with a lower cost than those of random networks ${ }^{27}$. In terms of these network properties, changes in cognitive functional status or cognitive capacity might be associated with changes in the configuration of brain functional networks ${ }^{26}$. Indeed, there are several lines of evidence suggesting that loss of the small-world configuration might be implicated in the cognitive deficits observed in various brain disorders, such as Alzheimer's disease, schizophrenia, and brain tumors ${ }^{28-30}$. Based on the aforementioned notion, our findings of changes in graph measures to high clustering and short path length after coffee consumption suggest that functional reorganization toward more efficient network properties might be a mechanism underlying the enhancement of cognitive function observed after coffee consumption. 
The mechanism underlying the shift in FC toward efficient network properties after coffee consumption remains to be determined. It is believed that caffeine's effect on cognition is associated with the blockade of the inhibitory properties of endogenous adenosine (particularly at A1 and A2A receptors), resulting in increased dopamine, norepinephrine, and glutamate release ${ }^{4}$. In addition, the cardiostimulatory effects of caffeine are considered to result from interactions with both adenosine and phosphodiesterase ${ }^{31}$. The caffeine-induced increases in dopamine and glutamate concentrations, coupled with phosphodiesterase inhibition, could be considered as a crucial mechanism underlying the net increase in the central nervous system and cardiovascular activity. Based on the actions of caffeine, it is plausible that the stimulatory effects of caffeine might directly lead to the reorganization of network properties toward a state of increased efficiency. Further studies are needed to unveil the mechanisms underlying the changes in network properties after coffee consumption.

Our findings of improved performance in the Digit Span Forward test suggest that attentional function could be enhanced by coffee consumption, which is in line with previous observations that coffee consumption has beneficial effects on attention ${ }^{7,32-34}$ In addition, our findings of greater performance in the Digit Span Backward test ${ }^{17}$ after coffee consumption may support findings from previous studies that have shown the role of coffee in improving working memory ${ }^{35-37}$. A recent functional magnetic resonance imaging ( $\mathrm{fMRI}$ ) study found that the alerting network, known as being responsible for maintaining an alert state throughout task performance, recruited a distributed network of brain regions, primarily the thalamus and bilateral fronto-parietal regions ${ }^{38,39}$. Based on these $\mathrm{fMRI}$ findings, our results that FC changes after coffee consumption are mainly observed in the fronto-centro-parietal regions imply that improvement of attentional function might be derived from activation of the alerting network.

We also found that performance in the Trail Making Test Part B was improved after coffee consumption and that the degree of improvement of the test was correlated with the changes in graph measures reflecting a shift toward more efficient network properties. It is well known that the Trail Making Test Part B is a representative tool for evaluating the ability of executive function responsible for psychomotor speed, visuospatial searching, target-directed motor tracking, and set-shifting ${ }^{40}$. Therefore, our findings further support previous studies that showed the beneficial effects of caffeine on executive function and psychomotor speed $^{4,5,41}$. Performance of executive controls requires activation of widespread prefrontal regions in concert with the anterior cingulate cortex ${ }^{4,42,43}$. These brain areas have been shown to be upregulated by caffeine ${ }^{37,44}$, supporting the stimulatory effects of caffeine on executive function. Moreover, dopamine was found to be a critical neurotransmitter for supporting executive function in these areas ${ }^{45}$. Given that dopamine concentrations can be increased by caffeine through blockade of the inhibitory properties of adenosine, caffeine may enhance executive function through the interaction of dopaminergic pathways with anterior cingulate and prefrontal cortical regions.

Our findings of the relationship between improved executive function and graph measures suggest that changing network topology toward more efficient network properties might be a crucial mechanism underlying the beneficial effects of coffee on executive function. Our speculation is supported by prior studies using fMRI that found increases in FC in multiple brain regions during the performance of the Trail Making Test Part $\mathrm{B}^{46-49}$. In addition, the aforementioned relationships were mainly observed in the alpha band, which is in accordance with a recent study showing that executive functions have a positive relationship with alpha coherence between regions of the right and left hemispheres ${ }^{50}$. Taken together, our findings support those of previous studies that coffee may enhance the FC responsible for performance on executive function, especially in the alpha band. Meanwhile, we did not find any changes in nodal graph measures after coffee consumption in the alpha band. The changes in global network properties without any region-specific changes in the alpha band suggest that coffee consumption might further enhance the improvement of physiological network efficiency responsible for activating cognitive function across the whole brain, rather than causing changes in the network properties of specific localized areas. Given the involvement of the dopaminergic pathways in executive function ${ }^{45}$, another plausible explanation is that our findings of changes in cortico-cortical network properties may not fully reflect the interactions of subcortical dopaminergic pathways with the cortical areas responsible for executive function.

We did not find any relationship between performance in Digit Span tests and graph measures. It is not fully understood why the results of the Digit Span tests, which reflect the function of attention/working memory, were not correlated with graph measures. However, it is plausible that there was a ceiling effect in the performance of the Digit Span tests in our cognitively normal 
population. In addition, given that attention/working memory is associated with not only cortical function but also various subcortical neurotransmitter systems (e.g., basal forebrain cholinergic systems and dopaminergic systems), a FC analysis evaluating the cortico-cortical network using EEG might not be sufficient to reveal the mechanism underlying the attention/working memory function.

There are several limitations of the present study that should be considered when interpreting our results. First, our study population was relatively small, and was only composed of highly educated young adults. Therefore, our results could not be generalized to the overall population, especially to the elderly. Second, we did not measure individual differences in biological susceptibility to caffeine or expectancy for coffee drinking to stimulate cognitive function ${ }^{11}$. Further studies incorporating measurements of caffeine blood level and investigation of a subjective expectation of coffee drinking as a cognitive enhancer may clarify the dose-response relationship and main contributor of the FC changes. Third, the results of the neuropsychological tests after coffee consumption may be biased due to learning effects. However, learning effects were likely mitigated by the use of different sets of contents in the repetition of the same tests. Finally, since canned coffee contains various ingredients other than caffeine, it is unclear whether our results were due to the effect of caffeine or the combined effects with other ingredients. Nevertheless, our study is the first EEG network analysis investigating the effects of canned coffee, containing a precisely controlled content of caffeine, on neurocognitive function.

The strength of our study is that FC was evaluated using two methods, coherence and PLI, which were compared to mitigate the limitations of scalp-level EEG analysis. We used two representative building blocks for characterizing brain FC in sensor space, coherence, and PLI, and obtained consistent results. Coherence is the most common method used to quantify the correlation between signals from different brain regions in terms of both amplitude and phase. In contrast, PLI measures the stability of the phase differences of short- and long-range neuronal activities over time independent of the amplitude of oscillations. This method is designed to reliably estimate phase synchronization against the presence of common sources such as volume conduction and active reference electrodes. In brief, it can be accomplished by discarding 0 and $\pi$ phase differences between two time series ${ }^{21}$.

\section{Conclusion}

Our results support the general belief and previous notion that coffee improves cognitive function. Moreover, our findings suggest that the beneficial effects of coffee might be attributed to reorganization of FC toward more efficient network properties. Our findings of changes in network properties may provide novel insights into the biological mechanisms underlying the beneficial effects of coffee on cognitive function. Furthermore, the patterns of network reorganization could be quantitative markers for elucidating the mechanisms underlying the beneficial effects of coffee on cognition, especially executive function.

\section{Declarations}

\section{Acknowledgements}

This work was supported by grants of Korea University Anam Hospital, Korea University College of Medicine (JBK, 01700351, K1922861, K2022991) and Korea Institute of Science and Technology (KIST) Institutional program with projects (KH, 2E30762, $2 \mathrm{~K} 02430$ ). We would like to thank the participants themselves, all of whom contributed greatly to the successful completion of this study.

\section{Author contributions}

Conceptualization: H.K., J.B.K.; Formal Analysis: S.H.K.(Soon Ho), K.H., J.B.K.; Data curation: S.H.K.(Sung Hoon), S.H.K.(Seong Hwan), J.H., and J.G.K.; Writing-original draft preparation: H.K., J.B.K.; Writing-review and editing: H.K., J.B.K.

\section{Competing interests}

The authors declare no competing interest.

\section{References}


1. Butt, M. S. \& Sultan, M. T. Coffee and its consumption: benefits and risks. Crit Rev Food Sci Nutr.51, 363-373 https://doi.org/10.1080/10408390903586412 (2011).

2. Ludwig, I. A., Clifford, M. N., Lean, M. E., Ashihara, H. \& Crozier, A. Coffee: biochemistry and potential impact on health. Food Funct.5, 1695-1717 https://doi.org/10.1039/c4fo00042k (2014).

3. Franke, A. G., Bagusat, C., Rust, S., Engel, A. \& Lieb, K. Substances used and prevalence rates of pharmacological cognitive enhancement among healthy subjects. Eur Arch Psychiatry Clin Neurosci.264 (Suppl 1), S83-90 https://doi.org/10.1007/s00406-014-0537-1 (2014).

4. Lorist, M. M. \& Tops, M. Caffeine, fatigue, and cognition. Brain Cogn.53, 82-94 https://doi.org/10.1016/s02782626(03)00206-9 (2003).

5. McLellan, T. M., Caldwell, J. A. \& Lieberman, H. R. A review of caffeine's effects on cognitive, physical and occupational performance. Neurosci Biobehav Rev.71, 294-312 https://doi.org/10.1016/j.neubiorev.2016.09.001 (2016).

6. Souissi, Y., Souissi, M. \& Chtourou, H. Effects of caffeine ingestion on the diurnal variation of cognitive and repeated highintensity performances. Pharmacology Biochemistry and Behavior.177, 69-74 (2019).

7. Einother, S. J. \& Giesbrecht, T. Caffeine as an attention enhancer: reviewing existing assumptions. Psychopharmacology (Berl).225, 251-274 https://doi.org/10.1007/s00213-012-2917-4 (2013).

8. Smith, A., Kendrick, A., Maben, A. \& Salmon, J. Effects of breakfast and caffeine on cognitive performance, mood and cardiovascular functioning. Appetite.22, 39-55 (1994).

9. Brunye, T. T., Mahoney, C. R., Lieberman, H. R., Giles, G. E. \& Taylor, H. A. Acute caffeine consumption enhances the executive control of visual attention in habitual consumers. Brain Cogn.74, 186-192 https://doi.org/10.1016/j.bandc.2010.07.006 (2010).

10. Edwards, S., Brice, C., Craig, C. \& Penri-Jones, R. Effects of caffeine, practice, and mode of presentation on Stroop task performance. Pharmacology Biochemistry and Behavior.54, 309-315 (1996).

11. Oei, A. \& Hartley, L. R. The effects of caffeine and expectancy on attention and memory. Hum Psychopharmacol.20, 193-202 https://doi.org/10.1002/hup.681 (2005).

12. Varela, F., Lachaux, J. P., Rodriguez, E. \& Martinerie, J. The brainweb: phase synchronization and large-scale integration. Nature reviews neuroscience.2, 229-239 (2001).

13. Friston, K. J. Functional and effective connectivity in neuroimaging: a synthesis. Human brain mapping.2, 56-78 (1994).

14. Sporns, O. Structure and function of complex brain networks. Dialogues Clin Neurosci.15, 247-262 (2013).

15. Farahani, F. V., Karwowski, W. \& Lighthall, N. R. Application of Graph Theory for Identifying Connectivity Patterns in Human Brain Networks: A Systematic Review. Front Neurosci.13, 585 https://doi.org/10.3389/fnins.2019.00585 (2019).

16. Rogers, P. J. Caffeine, mood and mental performance in everyday life. Nutrition Bulletin.32, 84-89 (2007).

17. Baddeley, A. The episodic buffer: a new component of working memory? Trends in cognitive sciences.4, 417-423 (2000).

18. Kortte, K. B., Horner, M. D. \& Windham, W. K. The trail making test, part B: cognitive flexibility or ability to maintain set? App/ Neuropsychol.9, 106-109 https://doi.org/10.1207/S15324826AN0902_5 (2002).

19. Fredholm, B. B., Battig, K., Holmen, J., Nehlig, A. \& Zvartau, E. E. Actions of caffeine in the brain with special reference to factors that contribute to its widespread use. Pharmacol Rev.51, 83-133 (1999).

20. Thatcher, R. W., Krause, P. J. \& Hrybyk, M. Cortico-cortical associations and EEG coherence: a two-compartmental model. Electroencephalogr Clin Neurophysiol.64, 123-143 https://doi.org/10.1016/0013-4694(86)90107-0 (1986).

21. Stam, C. J., Nolte, G. \& Daffertshofer, A. Phase lag index: assessment of functional connectivity from multi channel EEG and MEG with diminished bias from common sources. Hum Brain Mapp.28, 1178-1193 https://doi.org/10.1002/hbm.20346 (2007).

22. Fraga Gonzalez, G. et al. EEG Resting State Functional Connectivity in Adult Dyslexics Using Phase Lag Index and Graph Analysis. Front Hum Neurosci.12, 341 https://doi.org/10.3389/fnhum.2018.00341 (2018).

23. Rubinov, M. \& Sporns, O. Complex network measures of brain connectivity: uses and interpretations. Neuroimage.52, 10591069 https://doi.org/10.1016/j.neuroimage.2009.10.003 (2010). 
24. Mijalkov, M. et al. A graph theory software for the analysis of brain connectivity. PLoS One 12.BRAPH, e0178798 https://doi.org/10.1371/journal.pone.0178798 (2017).

25. Gramfort, A. et al. MEG and EEG data analysis with MNE-Python. Front Neurosci.7, 267 https://doi.org/10.3389/fnins.2013.00267 (2013).

26. Bassett, D. S. \& Bullmore, E. Small-world brain networks. Neuroscientist.12, 512-523 https://doi.org/10.1177/1073858406293182 (2006).

27. Simard, D., Nadeau, L. \& Kröger, H. Fastest learning in small-world neural networks. Phys. Lett. A.336, 8-15 (2005).

28. Stam, C. et al. Graph theoretical analysis of magnetoencephalographic functional connectivity in Alzheimer's disease. Brain.132, 213-224 (2009).

29. Liu, Y. et al. Disrupted small-world networks in schizophrenia. Brain.131, 945-961 https://doi.org/10.1093/brain/awn018 (2008).

30. Bosma, l. et al. Disturbed functional brain networks and neurocognitive function in low-grade glioma patients: a graph theoretical analysis of resting-state MEG. Nonlinear Biomed Phys.3, 9 https://doi.org/10.1186/1753-4631-3-9 (2009).

31. Davis, J. M. et al. Central nervous system effects of caffeine and adenosine on fatigue. Am J Physiol Regul Integr Comp Physiol.284, R399-404 https://doi.org/10.1152/ajpregu.00386.2002 (2003).

32. Alharbi, W. D. M., Azmat, A. \& Ahmed, M. Comparative effect of coffee robusta and coffee arabica (Qahwa) on memory and attention. Metabolic brain disease.33, 1203-1210 https://doi.org/10.1007/s11011-018-0230-6 (2018).

33. Haskell-Ramsay, C. F. et al. The Acute Effects of Caffeinated Black Coffee on Cognition and Mood in Healthy Young and Older Adults. Nutrients.10, https://doi.org/10.3390/nu10101386 (2018).

34. Brunye, T. T., Mahoney, C. R., Lieberman, H. R. \& Taylor, H. A. Caffeine modulates attention network function. Brain Cogn.72, 181-188 https://doi.org/10.1016/j.bandc.2009.07.013 (2010).

35. Klaassen, E. B. et al. The effect of caffeine on working memory load-related brain activation in middle-aged males. Neuropharmacology.64, 160-167 https://doi.org/10.1016/j.neuropharm.2012.06.026 (2013).

36. Nehlig, A. Is caffeine a cognitive enhancer? Journal of Alzheimer's disease: JAD.20 Suppl (1), S85-94 https://doi.org/10.3233/jad-2010-091315 (2010).

37. Koppelstaetter, F. et al. Does caffeine modulate verbal working memory processes? An fMRI study. Neuroimage.39, 492-499 https://doi.org/10.1016/j.neuroimage.2007.08.037 (2008).

38. Fan, J., McCandliss, B. D., Sommer, T., Raz, A. \& Posner M. I. Testing the efficiency and independence of attentional networks. J Cogn Neurosci.14, 340-347 https://doi.org/10.1162/089892902317361886 (2002).

39. Fan, J., McCandliss, B. D., Fossella, J., Flombaum, J. I. \& Posner, M. I. The activation of attentional networks. Neuroimage.26, 471-479 https://doi.org/10.1016/j.neuroimage.2005.02.004 (2005).

40. Arbuthnott, K. \& Frank, J. Trail making test, part B as a measure of executive control: validation using a set-switching paradigm. J Clin Exp Neuropsychol.22, 518-528 https://doi.org/10.1076/1380-3395(200008)22:4;1-0;FT518 (2000).

41. Soar, K., Chapman, E., Lavan, N., Jansari, A. S. \& Turner, J. J. Investigating the effects of caffeine on executive functions using traditional Stroop and a new ecologically-valid virtual reality task, the Jansari assessment of Executive Functions (JEF((c))). Appetite.105, 156-163 https://doi.org/10.1016/j.appet.2016.05.021 (2016).

42. Bush, G., Luu, P. \& Posner, M. I. Cognitive and emotional influences in anterior cingulate cortex. Trends Cogn Sci.4, 215-222 https://doi.org/10.1016/s1364-6613(00)01483-2 (2000).

43. Fan, J., Flombaum, J. I., McCandliss, B. D., Thomas, K. M. \& Posner, M. I. Cognitive and brain consequences of conflict. Neuroimage.18, 42-57 https://doi.org/10.1006/nimg.2002.1319 (2003).

44. Koppelstaetter, F. et al. Caffeine and cognition in functional magnetic resonance imaging. J Alzheimers Dis.20 Suppl (1), S71-84 https://doi.org/10.3233/JAD-2010-1417 (2010).

45. Ko, J. H. et al. Increased dopamine release in the right anterior cingulate cortex during the performance of a sorting task: a [11C]FLB 457 PET study. Neuroimage.46, 516-521 https://doi.org/10.1016/j.neuroimage.2009.02.031 (2009).

46. Moll, J., de Oliveira-Souza, R., Moll, F. T., Bramati, I. E. \& Andreiuolo, P. A. The cerebral correlates of set-shifting: an fMRI study of the trail making test. Arquivos de neuro-psiquiatria60, 900-905, doi:10.1590/s0004-282x2002000600002 (2002). 
47. Jacobson, S. C., Blanchard, M., Connolly, C. C., Cannon, M. \& Garavan, H. An fMRI investigation of a novel analogue to the Trail-Making Test. Brain and cognition.77, 60-70 https://doi.org/10.1016/j.bandc.2011.06.001 (2011).

48. Allen, M. D., Owens, T. E., Fong, A. K. \& Richards, D. R. A functional neuroimaging analysis of the Trail Making Test-B: implications for clinical application. Behavioural neurology.24, 159-171 https://doi.org/10.3233/ben-2011-0278 (2011).

49. Zakzanis, K. K., Mraz, R. \& Graham, S. J. An fMRI study of the Trail Making Test. Neuropsychologia.43, 1878-1886 https://doi.org/10.1016/j.neuropsychologia.2005.03.013 (2005).

50. Basharpoor, S., Heidari, F. \& Molavi, P. EEG coherence in theta, alpha, and beta bands in frontal regions and executive functions. App/ Neuropsychol Adult. 1-8 https://doi.org/10.1080/23279095.2019.1632860 (2019).

\section{Figures}

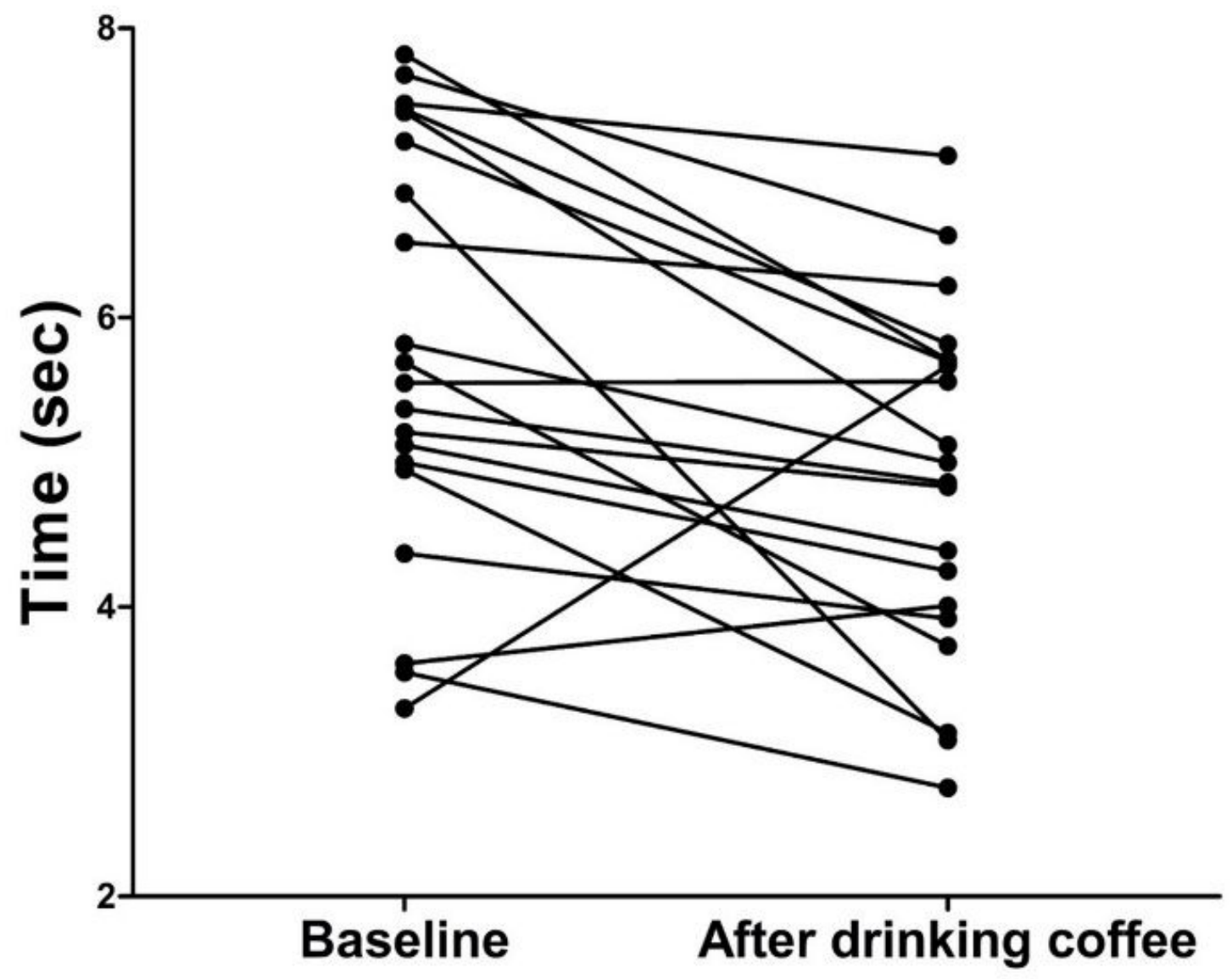

\section{Trail Making Test Part B}

\section{Figure 1}

Acute effects of coffee consumption on executive function. Individual changes in time (s) to complete Trail Making Test Part B are presented. 


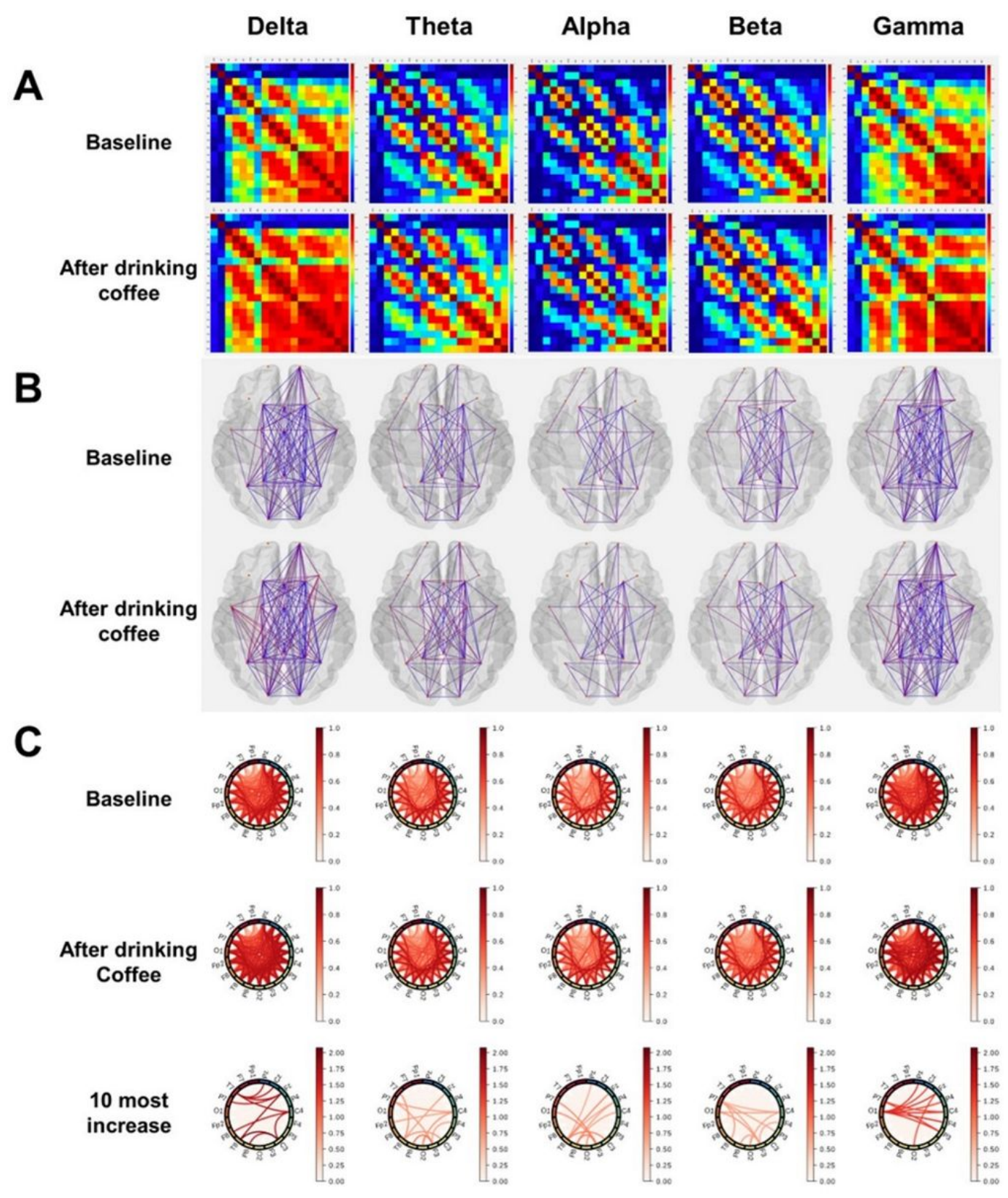

Figure 2

Coherence averaged across all subjects. (A) The plots show the coherence between 19 pairs of scalp electroencephalography electrodes in each frequency band at baseline (upper) and after coffee consumption (low). (B) Brain topologies of functional connectivity at baseline (upper) and after coffee consumption (low) are presented. (C) Each column corresponds to a frequency band as indicated on the top of the figure. Coherence matrices corresponding to the resting-state EEG before and after coffee consumption are plotted on the first and second rows, respectively. The third row plots the weight changes of the ten edges with the largest change. 


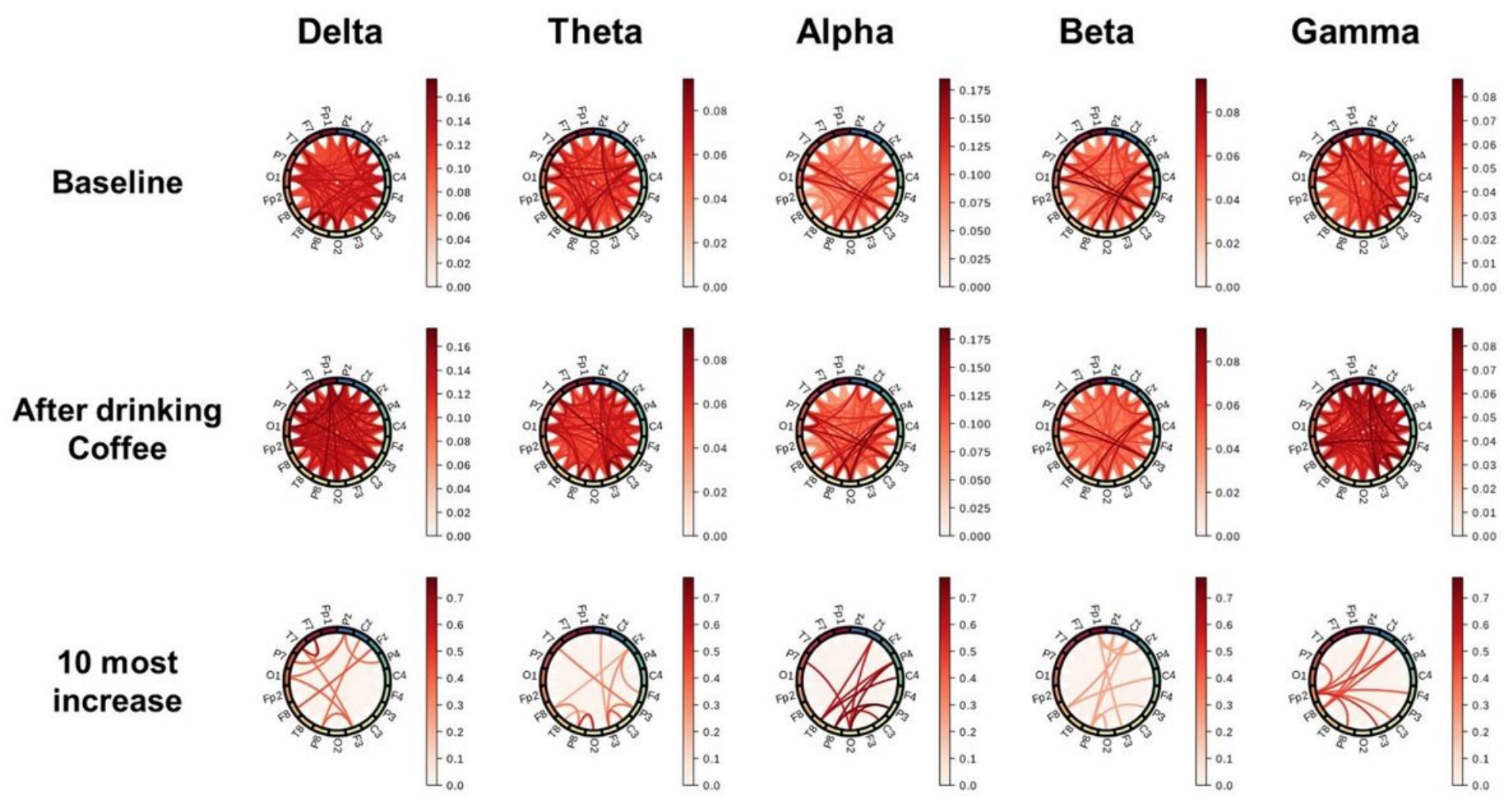

Figure 3

Phase lag index (PLI) averaged across all subjects. Each column corresponds to a frequency band as indicated on the top of the figure. PLI matrices corresponding to the resting-state EEG before and after coffee consumption are plotted on the first and second rows, respectively. The third row plots the weight changes of the ten edges with the largest change.

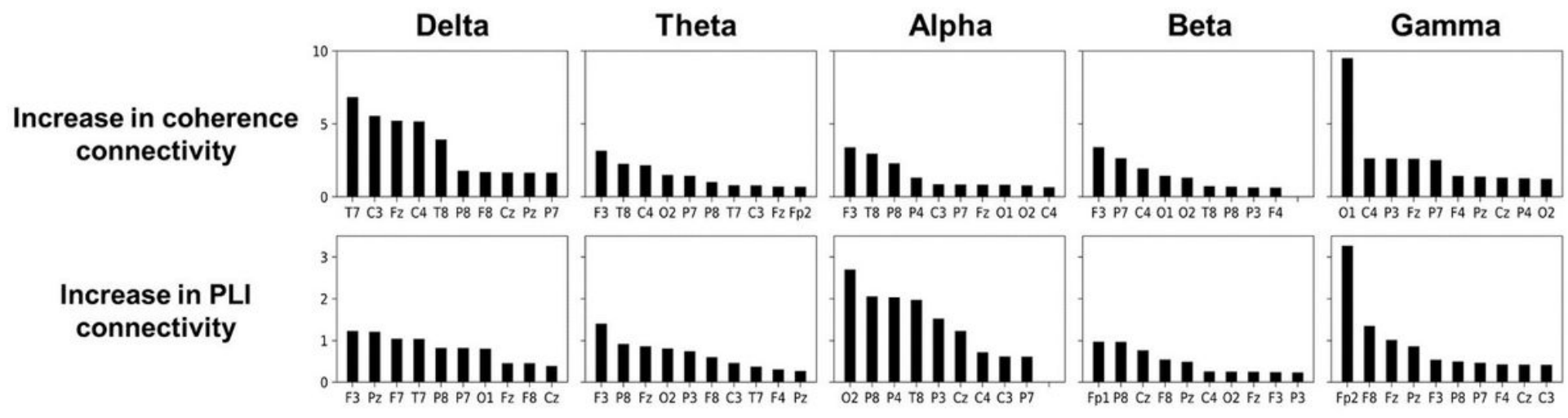

Figure 4

Ten most highly increased nodes in each band. The ten channels (nodes) with the highest increase in connectivity in terms of coherence (top row) and PLI (bottom row) are shown for each frequency band (corresponding to each column). Node connectivity was determined by taking the ten most highly increased links after coffee consumption from the averaged functional connectivity matrix (as in the third rows of Figures $2 \mathrm{C}$ and Figure 3 ) and summing the weights of those edges connected to each node. 
A

Delta

Theta

Alpha

Beta

Gamma
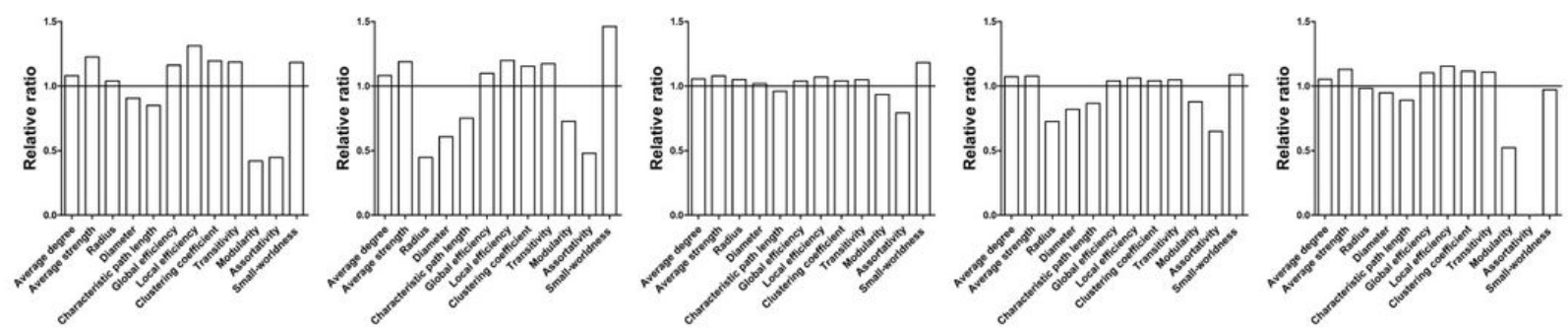

B

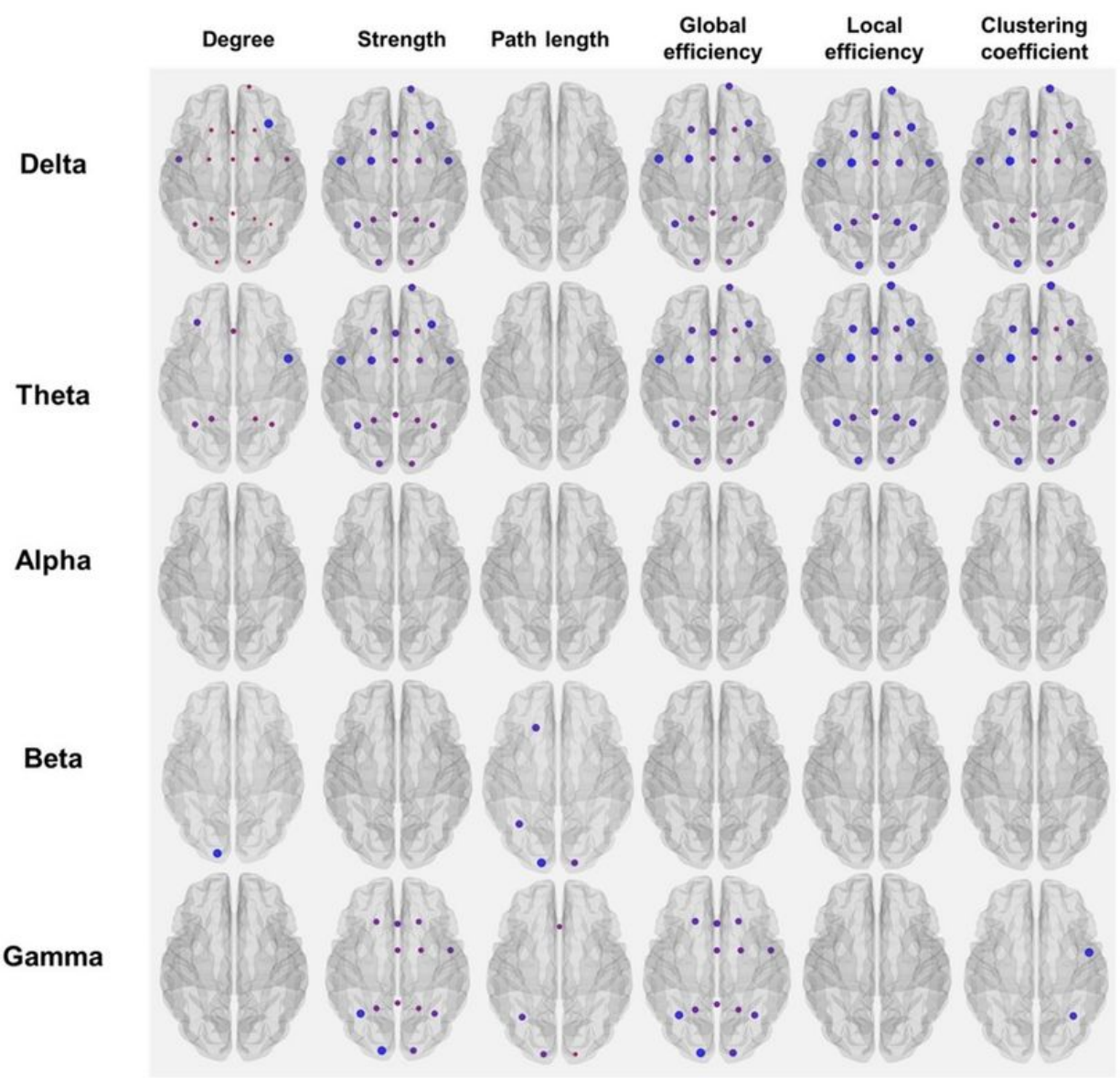

\section{Figure 5}

Results of graph theoretical analyses and correlation analysis. (A) By setting the values of graph measures at baseline to 1.0 (shown as a line at the value of 1.0), the relative ratio of global graph measures after coffee consumption are presented. (B) Changes after coffee consumption in the nodal measures of graph theoretical analyses are presented (FDR-corrected P $<0.05$ ). Larger nodes indicate greater differences between the conditions. The regions showing higher significance of differences are colored red, whereas the regions showing lower significance of difference are colored blue. 


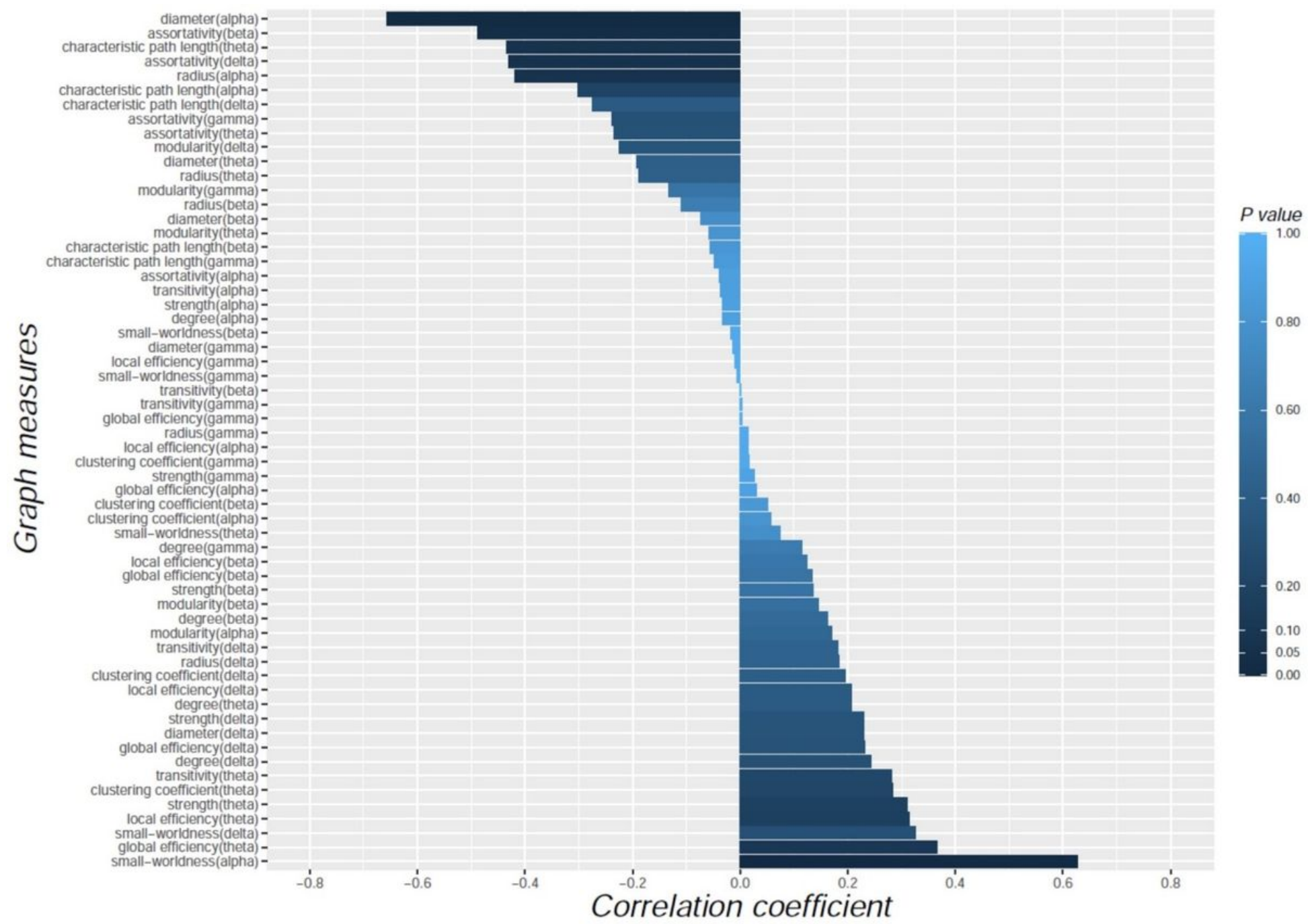

\section{Figure 6}

Correlations between the changes in global graph measures and the degree of improved performance on Trail Making Test Part B after coffee consumption. The horizontal bars represent the values of correlation coefficient. The color bar represents statistical significance ( $P$ value). 